\title{
Variaciones y continuidades: el uso de los recursos faunísticos en asentamientos indígenas del siglo $\mathrm{XX}$ en el centro-oeste de Santa Cruz
}

Agustín M. Agnolin*

\section{Resumen}

En este trabajo se propone un abordaje a las estrategias de uso de los recursos por parte de grupos familiares de origen indígena que habitaron la meseta del Strobel (Santa Cruz) durante el siglo XX. Dichos grupos se instalaron en la región evitando su incorporación a las reservas indígenas dispuestas por el Estado nacional. Como parte de la ocupación de este espacio, dichas unidades familiares implementaron diversas estrategias económicas, marcadas por el acomodamiento y reajuste ante el avance de la economía pastoril y la pérdida de tierras. Se concluye que las estrategias adoptadas se caracterizaron por su diversidad y flexibilidad, combinando prácticas tradicionales con aquellas vinculadas al nuevo lugar que estos grupos adquirieron en la economía. A pesar de ello, la aparición de nuevas legislaciones y el progresivo arrinconamiento de los grupos locales llevaron al abandono de la meseta por parte de éstos.

Variations and continuities: the use of animal resources during the $20^{\text {th }}$ century among indigenous settlements of central-western Santa Cruz
Recibido: 9 de marzo de 2018

Aceptado:

31 de agosto de 2018

\section{Palabras clave}

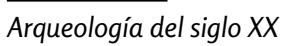
Grupos indígenas Pastoralismo Marginalidad Silencio histórico

Keywords

$20^{\text {th }}$ century archaeology Indigenous groups Pastoralism Marginality Historical silence 


\section{Introducción}

A fines del siglo XIX y comienzos del XX, la Patagonia es incorporada a los territorios controlados por el Estado Argentino, ocupando un nuevo rol en la economía regional (Bandieri, 2005; Barbería, 1987, 1996). En este proceso, la región se integra como un espacio principalmente destinado a la explotación ganadera, lo cual trajo aparejado la apropiación de las tierras disponibles por parte de propietarios particulares (Barbería, 1987, 1996). En este contexto, las poblaciones indígenas locales sufrieron un profundo proceso de reestructuración de sus características económicas y sociales (Goñi y Nuevo Delaunay, 2009; Martinic, 1995; Nuevo Delaunay, 2015). Estas poblaciones se vieron obligadas a habitar espacios cada vez menos productivos y alejados de las vías de comunicación (Barbería, 1987, 1996). Este fenómeno estableció un nuevo escenario para los grupos locales, caracterizado por un creciente arrinconamiento y pérdida de acceso a las tierras de caza, lo cual se vio acompañado del establecimiento de reservas estatales destinadas a la concentración de estos grupos (Barbería, 1987, 1996).

En este marco y de acuerdo a las informaciones etnográficas, las estrategias económicas de las poblaciones locales se caracterizaron hacia fines del siglo XIX y principios del $\mathrm{XX}$ por la combinación de la caza de guanacos y choiques con el comercio de cueros $\mathrm{y}$ pieles (Aguerre, 2000; Hatcher, 1903; Martinic, 1995). Estas estrategias requerían del uso de espacios amplios, los cuales resultaron más difíciles de hallar a partir del proceso de apropiación de tierras desarrollado en la región (Martinic, 1995).

Este proceso fue, sin embargo, diverso en términos espaciales y temporales. De este modo, algunos integrantes de estos grupos evitaron su confinamiento en reservas y se trasladaron a los sectores marginales carentes todavía de explotaciones ganaderas (Agnolin y Nuevo Delaunay, 2016; Goñi y Nuevo Delaunay, 2009; Nuevo Delaunay, 2007, 2012, 2013, 2015; Nuevo Delaunay, Goñi, Jimenez y Cecuk, 2014). Los asentamientos desarrollados en tierras aún no ocupadas por las estancias ganaderas afrontaron un conjunto de condiciones desfavorables, como la escasez de tierras y ambientes marcadamente estacionales, con limitada capacidad de carga animal (Nuevo Delaunay, 2015). Dichas condiciones implicaron que quienes recurrieron a estas modalidades de ocupación del espacio debieron reestructurar sus estrategias de obtención de recursos para lidiar con la nueva situación (Agnolin y Nuevo Delaunay, 2016; Goñi y Nuevo Delaunay, 2009; Nuevo Delaunay, 2015).

La modificación de los patrones de subsistencia no habría implicado en este caso un corte abrupto con las estrategias previas registradas etnográficamente, sino más bien una continuidad de prácticas tradicionales junto con la adopción de nuevos elementos, como el pastoreo de ovinos y caballos, la horticultura y el trabajo asalariado (Nuevo Delaunay, 2007). Tal panorama finaliza a mediados del siglo XX, cuando el establecimiento de una nueva legislación provincial para la tenencia de tierras limitó la explotación de los campos sobre los que no se poseían títulos de propiedad (Nuevo Delaunay, 2015).

En este contexto se enmarcan las tareas de investigación desarrolladas en los sitios Puesto Yatel y Puesto Quintillán, ubicados en la cuenca del lago Strobel (centro oeste de la Provincia de Santa Cruz) (Figura 1). Éstos fueron ocupados entre aproximadamente 1928/1940 y 1939/1968 respectivamente, por grupos familiares de ascendencia indígena (Casamiquela, Martinic, Mondelo y Perea, 1991). El contexto de ambas ocupaciones fue diferente, ya que Puesto Yatel fue habitado en un momento en el cual hubo una mayor disponibilidad de tierras, con las estancias más cercanas instaladas en el Lago Cardiel, a más de $30 \mathrm{~km}$. En el caso de la ocupación de Puesto Quintillán, la misma se inicia en un contexto similar, pero continúa durante la ocupación de la cuenca del Lago Strobel por parte de las estancias y la apropiación de gran parte de las tierras, alrededor de 1950 (Nuevo Delaunay, 2012). 


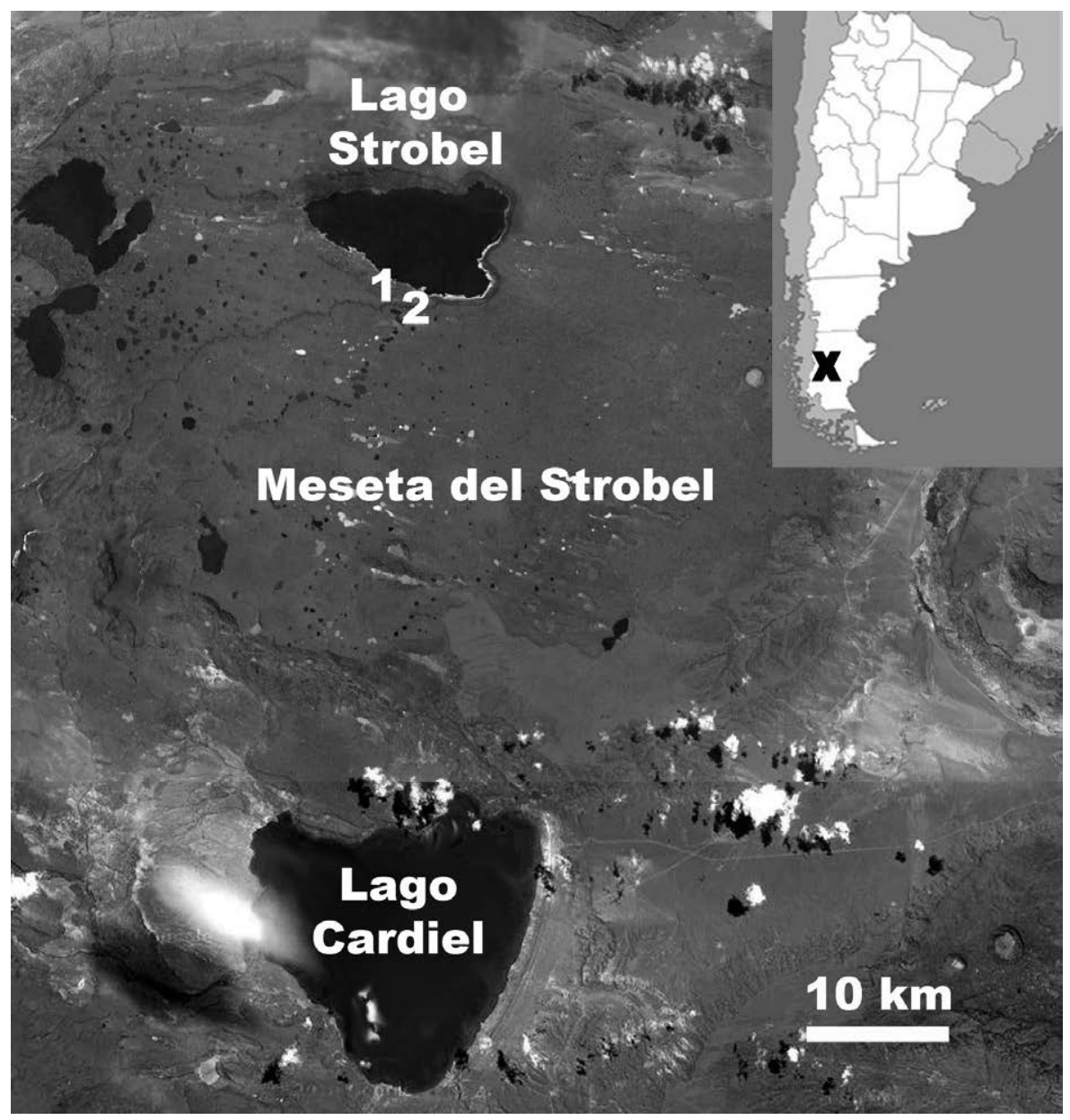

Figura 1. Mapa de la región de estudio con la ubicación de los sitios: 1) Puesto Quintillán, 2) Puesto Yatel.

Los sitios Puesto Yatel y Puesto Quintillán (PY y PQ de ahora en adelante), analizados en este trabajo han sido descriptos en trabajos previos en los que se abordaron aspectos de su registro arqueofaunístico, tecnología y estructuras (Agnolin y Nuevo Delaunay, 2016; Goñi y Nuevo Delaunay, 2009; Nuevo Delaunay, 2007, 2012, 2013, 2015; Nuevo Delaunay et al., 2014). El registro arqueofaunísico de los sitios ha sido analizado en un trabajo previo, abordando los indicios de comercio y el rol que ocuparon los diferentes taxones en las estrategias de subsistencia (Agnolin y Nuevo Delaunay, 2016). En este trabajo se discutirán los agentes actuantes en la formación de los depósitos arqueológicos y el procesamiento de los recursos faunísticos. El objeto del mismo es presentar nueva información referente a las alteraciones sufridas por los restos óseos, ya sea de origen antrópico o no, con el objeto de contribuir al conocimiento de un registro escasamente abordado por la arqueología patagónica. El resultado de estos análisis será discutido en el marco de los procesos sociales de arrinconamiento y marginalidad en los cuales actuaron los ocupantes de los sitios PY y PQ.

\section{Los sitios}

Los dos sitios analizados, PY y PQ, se encuentran ubicados dentro del Departamento de Río Chico de la provincia de Santa Cruz (Figura 1) en la meseta del Strobel, a orillas del lago homónimo. Esta meseta es un plateau basáltico de origen miocénico (Ramos, 1982) con alturas que oscilan en torno a los 700 y $1200 \mathrm{~m}$ s.n.m., cubierta por una 
vegetación de estepa arbustiva y herbácea (Morello, Matteucci, Rodríguez y Silva, 2012). Este espacio se caracteriza por un clima seco y marcadamente estacional, con un alto riesgo invernal (Oliva, González, Rial, Livraghi, 2001). Tales características ambientales, junto con su lejanía a las vías de comunicación, la convirtieron en un lugar tardíamente ocupado por las estancias ganaderas, las cuales se instalaron plenamente en la zona recién entrado el siglo XX (Nuevo Delaunay, 2012; Nuevo Freire y Vázquez Mercerat, 2014). Estas características incidieron en el abandono de parte de las estancias ubicadas en este sector de meseta tras la caída de los precios internacionales de la lana, a fines del siglo XX (Nuevo Delaunay, 2015).

En estudios previos se recopiló información oral y escrita sobre los sitios (Goñi y Nuevo Delaunay, 2009; Nuevo Delaunay, 2007, 2008, 2012, 2013, 2015). De acuerdo con dichas fuentes PY fue ocupado por 10 o 12 integrantes de la familia Yatel entre 1928 y 1940. Por otra parte, PQ fue ocupado por siete miembros de la familia Quintillán entre 1939 y 1968. En ambos casos los relatos dan cuenta de prácticas correspondientes a la cría de yeguarizos y ovejas y la caza comercial. En el caso de PQ, las fuentes describen la existencia de una pequeña huerta y la confección de fajas y quillangos para la venta. Para la familia Yatel las fuentes mencionan una importante cantidad de yeguarizos en manos de José Yatel en momentos previos a la ocupación del puesto (Aguerre, 2000).

Actualmente, ambos sitios presentan estructuras edilicias parcialmente desmoronadas, destinadas originalmente a habitaciones y corrales, combinadas en PY con tumbas y en PQ con canales y estructuras de cultivo (Agnolin y Nuevo Delaunay, 2016; Goñi y Nuevo Delaunay, 2009; Nuevo Delaunay, 2007, 2008, 2012, 2013, 2012, 2015). Los dos sitios poseen en sus alrededores amplios basurales con abundantes materiales faunísticos y artefactuales (Agnolin y Nuevo Delaunay, 2016; Nuevo Delaunay, 2007, 2008, 2012, 2013).

\section{Materiales y métodos}

Para la obtención de la muestra analizada en el presente trabajo se llevaron a cabo recolecciones y excavaciones en los basurales de ambos sitios. Las mismas consistieron en cada sitio en una recolección superficial general (alrededor de $1000 \mathrm{~m}^{2}$ en PY y $400 \mathrm{~m}^{2}$ en $\mathrm{PQ}$ ), dos recolecciones superficiales de $2 \times 2 \mathrm{~m}$ en las que se recogió todo el material faunístico y artefactual y dos sondeos de $0,5 \times 0,5 \mathrm{~m}$, a lo que se suma una excavación de $1 \times 0,5 \mathrm{~m}$ en PY, a los fines de verificar el potencial estratigráfico del basural.

El material faunístico recuperado fue analizado de acuerdo con los lineamientos generales definidos por Mengoni Goñalons (1999), que incluyen el cálculo del NISP, MNE, MAU\% y MNI de la muestra (Tabla 1). Debido a la presencia, si bien escasa, de huesos largos completos, los mismos se incluyeron en la Tabla 2 como ejemplares de diáfisis y epífisis proximal y distal, sumando un individuo a cada uno de estos campos.

En cuanto a los criterios seguidos en la clasificación de los taxones, se emplearon las categorías utilizadas por Klein (1976) en vista de su simplicidad y adecuación a la muestra observada.

En este trabajo se consideró una perspectiva tafonómica (Lyman, 1994). En este sentido y con el objeto de discutir la influencia de la atrición diferencial en los perfiles anatómicos relevados, se realizaron correlaciones estadísticas mediante el test de Spearman (considerando un nivel de significación del 5\%) entre los perfiles obtenidos en el taxón oveja y los valores de densidad ósea publicados por Lyman (1994). Si bien las técnicas empleadas por Lyman (1994) han recibido críticas (Lam, Pearson, Marean 


\begin{tabular}{|c|c|c|c|c|c|c|c|c|c|c|}
\hline \multirow[b]{2}{*}{ Taxón } & \multicolumn{5}{|c|}{ Puesto Yatel } & \multicolumn{5}{|c|}{ Puesto Quintillán } \\
\hline & $\begin{array}{c}\text { NISP } \\
\text { estratigrafía }\end{array}$ & $\begin{array}{c}\text { NISP } \\
\text { superficie }\end{array}$ & $\begin{array}{l}\text { NISP } \\
\text { total }\end{array}$ & NISP\% & MNI & $\begin{array}{c}\text { NISP } \\
\text { estratigrafía }\end{array}$ & $\begin{array}{c}\text { NISP } \\
\text { superficie }\end{array}$ & $\begin{array}{l}\text { NISP } \\
\text { total }\end{array}$ & NISP\% & MNI \\
\hline Ovis aries & 159 & 79 & 238 & 71,4 & 10 & 150 & 14 & 164 & 93,1 & 4 \\
\hline Lama guanicoe & 15 & 26 & 41 & 12,3 & 3 & 3 & 3 & 6 & 3,4 & 1 \\
\hline Equus ferus & 11 & 12 & 23 & 6,9 & 2 & o & 1 & 1 & 0,5 & 1 \\
\hline Bos taurus & 1 & 1 & 2 & 0,6 & 1 & o & 2 & 2 & 1,1 & 1 \\
\hline $\begin{array}{l}\text { Lepus } \\
\text { europaeus }\end{array}$ & 3 & 3 & 6 & 1,8 & 1 & $\mathrm{o}$ & o & $\mathrm{o}$ & o & $\mathrm{o}$ \\
\hline Edentata indet. & 3 & $\mathrm{o}$ & 3 & 0,9 & 1 & 1 & o & 1 & 0,5 & 1 \\
\hline Zaedius pichiy & 2 & $\mathrm{o}$ & 2 & 0,6 & 1 & o & o & o & o & o \\
\hline $\begin{array}{l}\text { Chloephaga } \\
\text { picta }\end{array}$ & o & 6 & 6 & 1,8 & 2 & o & o & o & o & 0 \\
\hline $\begin{array}{l}\text { Pterocnemia } \\
\text { pennata }\end{array}$ & 1 & 2 & 3 & 0,9 & 1 & 0 & $\mathrm{o}$ & o & $\mathrm{o}$ & $\mathrm{o}$ \\
\hline Rodentia indet. & 6 & $\mathrm{o}$ & 6 & 1,8 & 1 & 1 & o & 1 & 0,5 & 1 \\
\hline Cánido & 1 & 3 & 3 & 0,9 & 1 & 1 & 0 & 1 & 0,5 & 1 \\
\hline $\begin{array}{l}\text { Mamífero } \\
\text { pequeño }\end{array}$ & 2 & $\mathrm{o}$ & 2 & $\mathrm{o}$ & $\mathrm{o}$ & 2 & $\mathrm{o}$ & 2 & $\mathrm{o}$ & 0 \\
\hline $\begin{array}{l}\text { Mamífero } \\
\text { mediano } \\
\text { grande }\end{array}$ & 812 & 2 & 814 & $\mathrm{o}$ & o & 183 & o & 183 & o & $\mathrm{o}$ \\
\hline $\begin{array}{l}\text { Mamífero } \\
\text { grande }\end{array}$ & 14 & 3 & 17 & $\mathrm{o}$ & o & 4 & o & 4 & o & o \\
\hline $\begin{array}{l}\text { Indeterminado } \\
\text { (NID) }\end{array}$ & 1584 & 5 & 1589 & 0 & $\mathrm{o}$ & 509 & $\mathrm{o}$ & 509 & $\mathrm{o}$ & o \\
\hline Total & 2614 & 142 & 2756 & 100 & o & 854 & 20 & 874 & 100 & o \\
\hline
\end{tabular}

Tabla 1. Estructura taxonómica de la muestra. Referencias: indet.: indeterminado.

y Chen, 2003; Stahl, 1999), continúan siendo ampliamente utilizadas y son el conjunto de medidas de densidad mineral ósea de referencia para ovejas (Ioannidou, 2003; Symmons, 2005). En el taxón guanaco, las correlaciones estadísticas se llevaron a cabo con los valores de densidad mineral ósea publicados por Stahl (1999) y se utilizaron en la modalidad ajustada a la metodología de Lyman (Stahl, 1999, p. 353), dado que favorece las comparaciones con los otros taxones. En el caso del caballo, los valores de densidad mineral ósea fueron obtenidos de Lam y colaboradores (1999).

Asimismo, se relevaron las alteraciones en la superficie de los huesos, incluyendo marcas de carnívoros, roedores, raíces y alteraciones químicas, de acuerdo a los criterios provistos por Binford (1981) y Fisher (1995). En el caso de la meteorización, se consideró el conjunto de rasgos definido por Behrensmeyer (1978) y su clasificación en estadios. Por otra parte, se calculó el índice de fragmentación de acuerdo a los criterios definidos por Lyman (1994). Otro aspecto evaluado fue la termoalteración, la cual se evaluó mediante el relevamiento de los rasgos propuestos por Stiner y colaboradores (Stiner, Kuhn, Weiner y Bar-Yosef, 1995) y se clasificó en tres estadios de acuerdo a la metodología de Mengoni Goñalons (1999).

En cuanto al procesamiento, se relevó el conjunto de acuerdo a los criterios provistos por la bibliografía para el análisis de marcas (Binford, 1981; Gifford-Gonzalez, 1989; Mengoni Goñalons, 1999; Potts y Shipman, 1981). Se consideró su tipo (percusión, machacado, raspado y corte) y se compararon sus características. Éstas consistieron, en el caso de las marcas de percusión, en su ubicación en la topografía del hueso y, en el caso de las 
marcas de corte, su cantidad, ubicación, largo y orientación, de acuerdo a la metodología sintetizada por Mengoni Goñalons (1999). Estos rasgos fueron comparados con las bases de datos de marcas disponibles con el objetivo de discutir su origen (Abe, 2005; Binford, 1981; Gifford-Gonzalez, 1989). Debe aclararse que, si bien estos corpus de datos se han obtenido entre sociedades de cazadores recolectores, se considera que los mismos se encuentran fuertemente constreñidos por la anatomía de las carcasas. De este modo, se espera cierta uniformidad en la función de dichos rasgos y por ende se considera que son factibles de ser interpretados de acuerdo a los marcos de referencia conocidos (Binford, 1981).

Por último, se consideró la relación entre los perfiles de representación de partes y los índices de utilidad económica. Los índices considerados fueron los de carne, médula, secado y utilidad general determinados para oveja por Binford (1978) y para el caballo por Outram y Rowley-Conwy (1997). En este último caso el índice de secado de carne fue el empleado por Binford (1978) para el caribú, debido a la inexistencia de un índice específico para este taxón. En el caso del guanaco, se emplearon los índices de secado de carne confeccionados por De Nigris y Mengoni Goñalons (2005), el de carne elaborado por Borrero (1990) y el de médula de Mengoni Goñalons (1999).

\section{Resultados}

\section{Aspectos tafonómicos}

En primer lugar, se buscó evaluar el peso que la atrición diferencial pudo tener en el registro arqueofaunístico de los puestos. De este modo, se aplicó el test de Spearman sobre los perfiles anatómicos de la oveja, el caballo y el guanaco (Tabla 2), en PY y de la oveja en PQ (Tabla 2). El resto de los taxones fueron excluidos debido a los pequeños tamaños de muestra que poseen. En todos los casos la correlación entre la densidad mineral ósea y el MAU\% arrojó resultados débiles y no significativos al nivel de 0,05 (Tabla 3). Dichos resultados indican que los sesgos en el perfil de representación de partes esqueletarias no son completamente atribuibles a la actividad de factores atricionales. A pesar de ello, debe remarcarse que la mayor parte de los elementos con una baja densidad ósea, como costillas y vértebras, presentan una escasa representación en la muestra (Agnolin y Nuevo Delaunay, 2016).

Las frecuencias de los estadios de meteorización en la muestra total de huesos no termoalterados se indican en la Tabla 4. La muestra se segmentó de acuerdo a la proveniencia de los materiales, con el objeto de poder evaluar la incidencia diferencial de la meteorización en los conjuntos de superficie y estratigrafía. De acuerdo con la información presentada, los especímenes se encuentran en su mayoría en estadios iniciales de meteorización, con las categorías 0,1 y 2 en torno al 80\%. Dentro de esto, se presentan diferencias en su preservación, estando el material proveniente de superficie más meteorizado, con más de la mitad de sus especímenes en estadio 2 en ambos sitios.

Un aspecto destacable es que los huesos de caballo presentan una mayor frecuencia de estadios más avanzados de meteorización. En este sentido, el patrón de meteorización de caballo en PY presenta un 66,6\% en los estadios 0, 1 y 2. Tal patrón podría estar vinculado al enterramiento diferencial de los elementos de menor tamaño, quedando los voluminosos huesos de este taxón expuestos por más tiempo. Asimismo, su mayor volumen podría estar incidiendo en las mayores posibilidades de identificabilidad de estos restos, incluso en el caso de huesos altamente fragmentados o meteorizados.

Se relevaron una serie de marcas producidas por carnívoros, cuyas características implican la existencia de una moderada a baja intensidad de daño en los conjuntos (Tabla 5). 


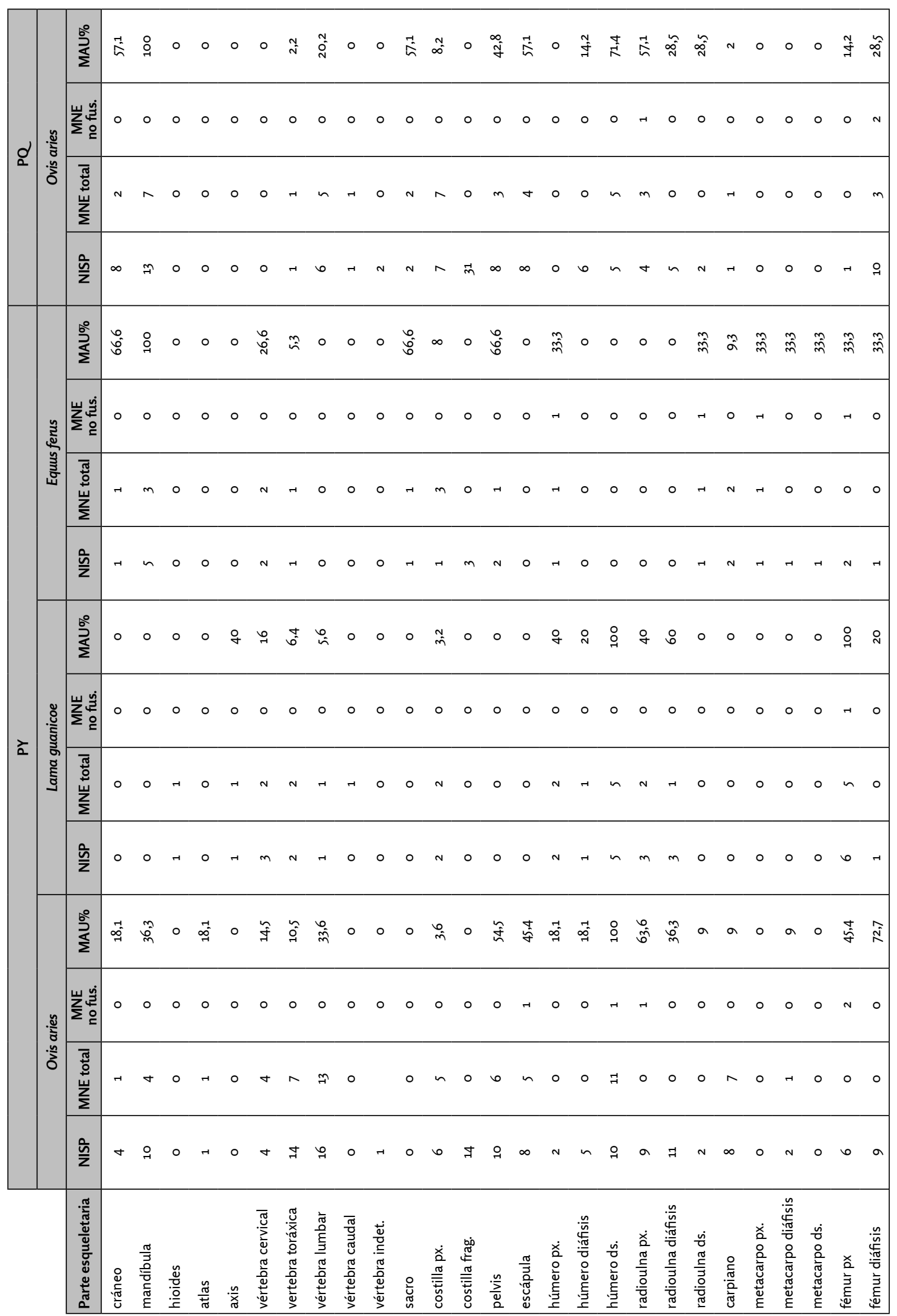

Tabla 2. NISP, MNE total, MNE de huesos no fusionados y MAU\% en oveja, caballo y guanaco de PY y oveja de PQ. Referencias: px.: proximal, ds.: distal, indet.: indeterminado, no fus.: elemento no fusionado, frag.: fragmento (continúa en página siguiente). 


\begin{tabular}{|c|c|c|c|c|c|c|c|c|c|c|c|c|c|c|c|c|c|c|}
\hline \multirow{4}{*}{$d$} & \multirow{4}{*}{ 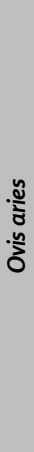 } & 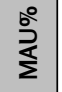 & $\circ$ & ₹ & 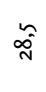 & $\overrightarrow{5}$ & $i$ & $\stackrel{\widehat{\alpha}}{\text { N }}$ & $\stackrel{\sim}{\widehat{\sim}}$ & ₹ & $\circ$ & 年 & $\circ$ & $\hat{\tilde{A}}$ & 궁 & $\dot{m}_{n}^{ \pm}$ & 0 & $\stackrel{H}{m}$ \\
\hline & & 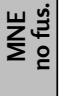 & $\circ$ & 4 & $\circ$ & -1 & 0 & $\circ$ & $\circ$ & $\circ$ & $\circ$ & $\circ$ & $\circ$ & $\circ$ & $\circ$ & $\circ$ & $\circ$ & $\circ$ \\
\hline & & 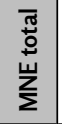 & $\circ$ & $\circ$ & $\circ$ & $\nabla$ & $N$ & $N$ & $N$ & - & 0 & 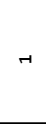 & 0 & n & $N$ & -1 & 0 & $N$ \\
\hline & & $\frac{\text { जे }}{z}$ & 0 & - & $m$ & $n$ & $N$ & $N$ & $N$ & - & 0 & N & $\circ$ & $n$ & $N$ & H & $N$ & $N$ \\
\hline \multirow{12}{*}{ え } & \multirow{4}{*}{ 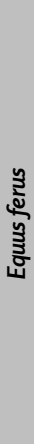 } & $\stackrel{\stackrel{\circ}{2}}{\stackrel{5}{\Sigma}}$ & $\circ$ & $\circ$ & $\begin{array}{l}0 \\
b^{0}\end{array}$ & $\circ$ & $\begin{array}{l}b^{\circ} \\
b^{-}\end{array}$ & $\stackrel{\text { m }}{\text { ñ }}$ & $\circ$ & 0 & 0 & 0 & 0 & $\circ$ & $\circ$ & 0 & $\circ$ & 0 \\
\hline & & 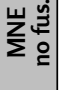 & $\circ$ & $\circ$ & $\circ$ & $\circ$ & $\circ$ & - & $\circ$ & $\circ$ & $\circ$ & 0 & $\circ$ & $\circ$ & $\circ$ & $\circ$ & $\circ$ & 0 \\
\hline & & $\begin{array}{l}\overline{\widetilde{J}} \\
\stackrel{0}{ \pm} \\
\text { 崩 }\end{array}$ & 0 & $\circ$ & $N$ & $\circ$ & 0 & $r$ & 0 & 0 & 0 & 0 & $\circ$ & 0 & $\circ$ & 0 & $\circ$ & 0 \\
\hline & & 㐫 & $\circ$ & $\circ$ & $m$ & 0 & $N$ & - & $\circ$ & $N$ & 0 & 0 & $\circ$ & $\circ$ & $\circ$ & $\circ$ & 0 & 0 \\
\hline & \multirow{4}{*}{ 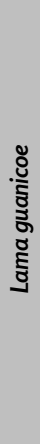 } & $\stackrel{\circ}{\stackrel{\circ}{\Sigma}}$ & ํ. & 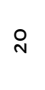 & 요 & $\circ$ & $\circ$ & กั & $\stackrel{\text { N }}{\text { s. }}$ & $\circ$ & $\circ$ & ㅇ & 0 & $\stackrel{\text { N }}{n^{2}}$ & $\circ$ & $\circ$ & 0 & $\circ$ \\
\hline & & 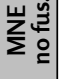 & $\circ$ & $\circ$ & $\circ$ & $\circ$ & $\circ$ & $\circ$ & $\circ$ & 0 & $\circ$ & 0 & 0 & $\circ$ & $\circ$ & 0 & $\circ$ & 0 \\
\hline & & 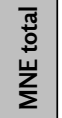 & 0 & -4 & $\circ$ & $\circ$ & $\circ$ & - & -1 & $\circ$ & $\circ$ & - & 0 & - & $\circ$ & 0 & 0 & 0 \\
\hline & & $\frac{\grave{n}}{z}$ & $r$ & - & $r$ & $\circ$ & $\circ$ & - & - & $\circ$ & 0 & - & $\circ$ & - & $\circ$ & 0 & $\circ$ & $\circ$ \\
\hline & \multirow{4}{*}{ 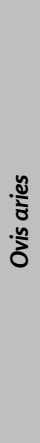 } & $\stackrel{\circ}{\stackrel{5}{\Sigma}}$ & $\circ$ & $a$ & 索 & $\begin{array}{l}\infty \\
\text { స్ }\end{array}$ & $\widehat{\hat{N}}$ & $\hat{\omega}^{m}$ & $\hat{\nu}_{\tilde{n}}^{n}$ & 0 & 孜 & $a$ & 占 & 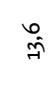 & $\tilde{F}$ & 年 & 0 & $\tilde{n}$ \\
\hline & & 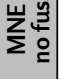 & $\circ$ & - & $\circ$ & n & 0 & $\circ$ & - & $\circ$ & 0 & $\circ$ & $N$ & m & - & $\circ$ & 0 & $\circ$ \\
\hline & & 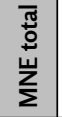 & $\circ$ & $\circ$ & 0 & $a$ & $\wedge$ & $\nabla$ & $\sigma$ & 0 & 0 & $\circ$ & $\infty$ & 6 & $n$ & $N$ & 0 & $m$ \\
\hline & & $\frac{\text { जि }}{z}$ & 0 & - & 우 & 엄 & $\infty$ & $\infty$ & $\infty$ & 0 & -1 & 6 & $\infty$ & $a$ & 6 & $N$ & $r$ & $m$ \\
\hline & & 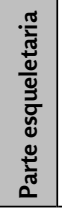 & 西 & 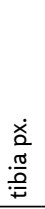 & 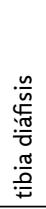 & 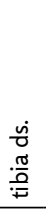 & 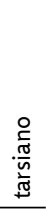 & 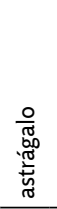 & 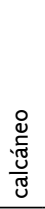 & 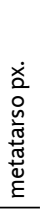 & 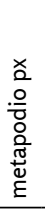 & 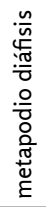 & 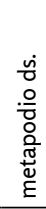 & 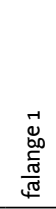 & 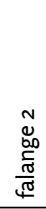 & 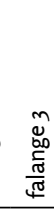 & 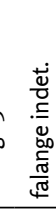 & 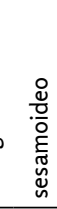 \\
\hline
\end{tabular}




\begin{tabular}{|c|c|c|c|}
\cline { 2 - 4 } \multicolumn{1}{c|}{} & Taxón & $\mathbf{R}$ & $\mathbf{p}$ \\
\hline \multirow{3}{*}{$\mathrm{PY}$} & Ovis aries & 0,12 & 0,5 \\
& $\begin{array}{c}\text { Equus ferus } \\
\text { Lama } \\
\text { guanicoe }\end{array}$ & 0,26 & 0,29 \\
\hline $\mathrm{PQ}$ & Ovis aries & 0,01 & 0,94 \\
\hline
\end{tabular}

Tabla 3. Resultados de la correlación entre el MAU\% y la densidad ósea para diversos taxones.

\begin{tabular}{|c|c|c|c|c|c|c|}
\hline Sitio & Proveniencia & $\mathbf{0}$ & $\mathbf{1}$ & $\mathbf{2}$ & $\mathbf{3}$ & $\mathbf{4}$ \\
\hline $\begin{array}{c}\text { Puesto } \\
\text { Yatel }(\mathrm{n}= \\
359)\end{array}$ & Superficie & $4,4(5)$ & $15,9(18)$ & $64,6(73)$ & $15,1(17)$ & 0 \\
\hline $\begin{array}{c}\text { Puesto } \\
\text { Quintillán }\end{array}$ & Superficie & $20,1(4)$ & $20,1(4)$ & $52,6(10)$ & $5,2(1)$ & 0 \\
$(\mathrm{n}=388)$ & Estratigrafía & $62,8(232)$ & $18,9(70)$ & $12,1(45)$ & $5,6(21)$ & $0,2(1)$ \\
\hline
\end{tabular}

Tabla 4. Distribución de porcentajes de estadios de meteorización. Entre paréntesis figura el $N$ correspondiente.

\begin{tabular}{|l|c|c|c|c|c|c|c|}
\hline Sitio & $\begin{array}{c}\text { NISP\% con } \\
\text { marcas }\end{array}$ & $\begin{array}{c}\text { NISP\% con } \\
\text { Punctures }\end{array}$ & $\begin{array}{c}\text { NISP\% con } \\
\text { Furrows }\end{array}$ & $\begin{array}{c}\text { NISP\% con } \\
\text { Scoring }\end{array}$ & $\begin{array}{c}\text { NISP\% con } \\
\text { Crenulated }\end{array}$ & $\begin{array}{c}\text { NISP\% } \\
\text { Crenulated y } \\
\text { Puncture }\end{array}$ & $\begin{array}{c}\text { NISP\% } \\
\text { Scoring y } \\
\text { Puncture }\end{array}$ \\
\hline $\begin{array}{l}\text { Puesto Yatel } \\
(\mathrm{n}=202)\end{array}$ & $16,3(33)$ & $14,8(30)$ & 0 & $0,4(1)$ & 0 & $0,9(2)$ & 0 \\
$\begin{array}{l}\text { Puesto Quintillán } \\
(\mathrm{n}=156)\end{array}$ & $22,7(34)$ & $19,2(30)$ & $0,6(1)$ & $0,6(1)$ & 0 & $0,6(1)$ & $0,6(1)$ \\
\hline
\end{tabular}

Tabla 5. Frecuencias y tipos de daño producido por carnívoros en cada sitio entre los especímenes identificados a nivel de especie. Entre paréntesis se indica el $\mathrm{N}$ correspondiente. En la segunda columna se consideran todos los especímenes con marcas de carnívoros sobre el total identificado.

El porcentaje de especímenes con marcas de carnívoros entre los materiales identificados a nivel de especie en PY es superior al 15\% y al 20\% en PQ. En ambos sitios, la distribución del daño se halla concentrada principalmente en los especímenes pertenecientes a oveja. Hay una amplia gama de tipos de daño presentes, siendo los punctures absolutamente mayoritarios, seguidos en menor proporción por furrowing, crenulated y scoring (Binford, 1981). En el caso de los punctures, se midió el diámetro en una muestra escogida al azar de diez ejemplares en cada conjunto, lo cual dio diámetros ubicados entre los 3 y $4 \mathrm{~mm}$. Por otra parte, los otros tipos de daño presentes resultan minoritarios, ya que el furrowing se presenta sólo en dos especímenes en huesos largos de PY y uno en un hueso axial en PQ. En consonancia con esto, sólo existe un ejemplar de cilindro óseo, presente en PQ. Esto, combinado con las evidencias que indican que la atrición diferencial no fue la que determinó el carácter del conjunto, llevan a considerar que el daño, si bien presente, no ha tenido un carácter extensivo o la suficiente intensidad como para alterar significativamente los perfiles anatómicos de la muestra.

Otro aspecto a tener en cuenta es el agente causante de los daños registrados. En este sentido, debe considerarse la existencia en los momentos estudiados de perros domésticos. Se infiere, de acuerdo a esto, que los perros han sido los principales causantes de estas modificaciones en las superficies del conjunto óseo, si bien esto 
no excluye la participación minoritaria de otros taxones. La presencia de perros en las estancias actuales en la región es ubicua, ya que resultan fundamentales como animales auxiliares para las tareas pastoriles. Asimismo, su utilización por parte de los grupos indígenas de los siglos XIX y XX está documentada en numerosas fuentes históricas, las que describen su uso frecuente como animal de caza y su empleo en el pastoreo (Martinic, 1995). La existencia de este animal está documentada no sólo por las fuentes orales y escritas, sino también por el tipo y la intensidad del daño presente en la muestra. En este sentido, el rango de tamaños de 3-4 $\mathrm{mm}$ de diámetro en los punctures ha sido considerado como el típico de los perros domésticos (si bien existe cierto grado de solapamiento entre carnívoros, ver entre otros, Andrés, Gidna, Yravedra y Domínguez-Rodrigo, 2012; Domínguez-Rodrigo y Piqueras, 2003; Haynes, 1980).

Por otra parte, la incidencia de roedores y raíces en la muestra ha resultado muy escasa. En este sentido, los porcentajes de marcas de roedores se encuentran en torno al 1\% del NISP en ambos sitios, mientras que las marcas de raíz en PQ alcanzan el $2 \%$ y sólo se ubican en los especímenes obtenidos en estratigrafía. En el caso de PY, las marcas de raíz se encuentran en torno al 1,3\% del NISP, estando algunas de ellas en el material recolectado en superficie. Este hecho indica que parte del material registrado en superficie estuvo enterrado en algún momento de su historia y fue posteriormente descubierto.

\section{Termoalteración}

La termoalteración en los conjuntos arqueofaunísticos puede deberse a varios motivos, tales como los métodos de cocción (Kent, 1993), la quema de los restos óseos como residuos (Asmussen, 2009; Walters, 1988) o a su uso como combustible (Costamagno, Griggo y Mourre, 1998; Costamagno, Thery-Parisot, Brugal y Guibert, 2006; TheryParisot, 2002). En este sentido, su evaluación resulta una vía de interés para abordar el uso de los recursos faunísticos en este caso.

Un aspecto que se destaca en la fauna de los sitios es el hecho de que presenta una alta frecuencia de especímenes termoalterados (Figura 2). Si consideramos la totalidad de la muestra, más del $80 \%$ de los especímenes están calcinados. Tales cifras superan ampliamente las generadas en procesos de cocción registrados etnográficamente (Kent, 1993), los que se acercan al 1\%. Por otra parte, la presencia mayoritaria del calcinado como tipo de daño indica que los especímenes fueron sometidos a temperaturas altas durante períodos prolongados (Lyman, 1994; Stiner et al., 1995).

Por otra parte, la distribución de la termoalteración en el conjunto resulta generalizada, estando todos los elementos esqueletarios afectados por el fuego. A pesar de que una amplia diversidad de partes muestra alteraciones por efecto de la combustión, existen algunas regiones como el esqueleto axial con una incidencia menor $(13,2 \%$ en PQ y $39,5 \%$ en PY). Tal distribución contrasta con lo esperado en el caso de la utilización de los huesos como combustible, en el que se espera que el mismo se concentre en las epífisis de huesos largos, costillas y vértebras (Costamagno et al., 1998, 2006; TheryParisot, 2002; Thery-Parisot, Brugal, Costamagno y Guilbert, 2004). Asimismo, la distribución de las marcas de combustión en las superficies de los huesos indica que muchos de ellos fueron quemados luego de ser fragmentados. En este sentido, en el caso de los huesos largos, hay ejemplares con las superficies de su canal medular afectadas por la combustión.

De este modo, la elevada frecuencia de termoalteración de la muestra habría incidido en su nivel de identificabilidad. En este sentido, si consideramos la frecuencia de termoalteración entre los especímenes identificados, es mucho menor que en la totalidad de la muestra (12,06\% en PQ y $41,1 \%$ en PY). 


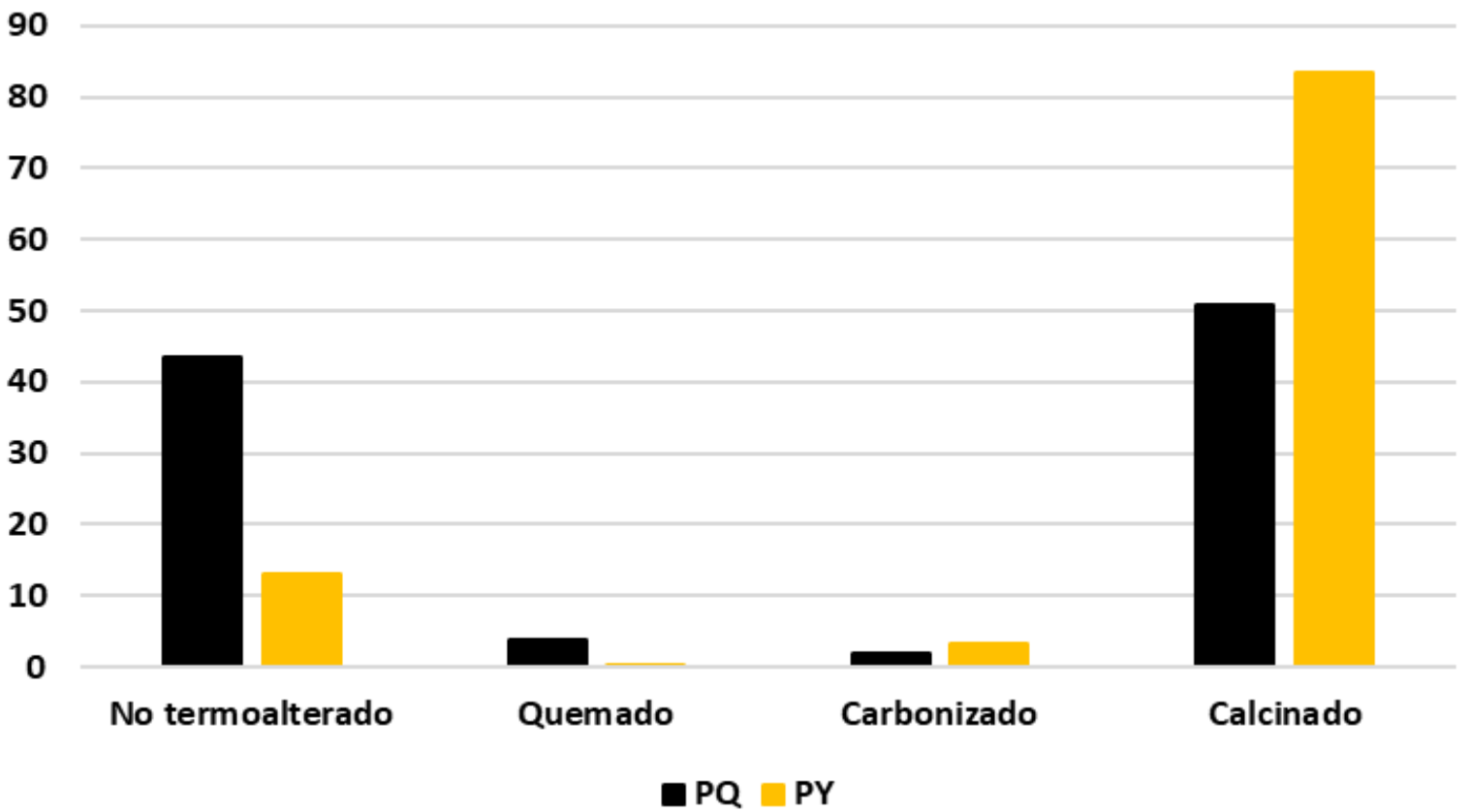

Figura 2. Especímenes afectados por el fuego en cada sitio (expresado en \%). Se consideró la totalidad de la muestra.

\section{Procesamiento y perfiles de representación de partes}

En ambas muestras las marcas de procesamiento se destacan por su abundancia y diversidad. En PY los taxones que presentan marcas son: oveja, caballo, guanaco, vaca, cánido, cauquén, liebre y choique. En el caso de los edentados, existen placas termoalteradas, las cuales son consideradas indicadores de su procesamiento, si bien también podrían ser fruto de la termoalteración generalizada que presenta el conjunto. Por su parte, los roedores no exhiben ningún tipo de marca, indicando un posible origen natural.

En PQ, las especies que poseen marcas son oveja, guanaco y vaca. Los roedores, edentados y cánidos no presentan ninguna marca. Entre los materiales identificados como edentados y cánidos, la ausencia de indicios de procesamiento podría deberse al escaso tamaño de muestra.

En ambos sitios, las marcas habrían sido producidas principalmente mediante el empleo de cuchillos y hachas de metal. Si bien en el primero de los sitios se han hallado artefactos líticos, es probable que los mismos pertenezcan a una ocupación previa (Nuevo Delaunay, 2008).

Las marcas relevadas fueron comparadas con los corpus documentados de alteraciones antrópicas en huesos (Abe, 2005; Binford, 1981; Gifford-Gonzalez, 1989), con el objeto de profundizar en el conocimiento de los patrones de procesamiento de las carcasas. De acuerdo con ello, en PY se han relevado marcas diversas para la oveja, con similares proporciones en elementos axiales y apendiculares. Dentro de este taxón predominan las huellas de corte seguidas por las de percusión, esta últimas únicamente en la región apendicular. Asimismo, existen ejemplos de machacado segmentando vértebras, pelvis y costillas. Las marcas indican un procesamiento primario y secundario, con extracción de carne y médula y segmentación de partes, posiblemente para ajustar su tamaño al de los recipientes de cocción. En el guanaco, las huellas son abundantes, especialmente las de corte y percusión, concentradas en el esqueleto apendicular. Estas indican obtención de médula y carne, así como segmentación de partes en unidades menores. Para el 
caballo, la mayoría de las marcas se ubican en el esqueleto axial, siendo mayoritarias las de corte y machacado. Las mismas se vinculan con tareas de descarne, desarticulación de partes y aprovechamiento de médula. Una de las marcas se ubica en la pelvis, la cual fue segmentada en dos partes mediante un golpe, posiblemente para ajustarla a un recipiente para su cocción. Entre los restos de cánido, una tibia distal posee rastros de corte, cuya ubicación los hace asignables al tipo Td-4 de Binford (1981), relacionado con el descarne de la pieza. Entre los escasos materiales asignados a vaca, existen huellas en una escápula vinculadas con el descarne y su fragmentación, posiblemente con el objeto de reducir su tamaño. En el caso del cauquén las marcas se interpretan como destinadas a la separación de las manos y patas del tronco, posiblemente como parte de su preparación para el consumo. En la liebre, si bien la muestra es chica, presenta una amplia diversidad, vinculada con la desarticulación, descarne e incluso un posible evento de aprovechamiento de médula. Asimismo, de los materiales asignados a choique, uno de ellos presenta marcas de corte y otro de percusión.

En PQ, la muestra ósea presenta una considerable representación de huellas de procesamiento. En este sentido, los materiales asignados a oveja presentan la mayor abundancia. Estas se centran en el esqueleto axial, predominando las de corte y seguidas por las de machacado, raspado y percusión. Estas últimas se ubican en los huesos largos, pero a diferencia de PY también en las mandíbulas, destinadas a la extracción de la médula ubicada bajo el tercer molar. Dichas huellas indican el procesamiento integral de las carcasas, con evidencias de descarne, extracción de médula, desarticulación y segmentación de partes para su ajuste a recipientes de cocción (en especial costillas y pelvis). El guanaco, si bien cuenta con una muestra escasa, posee indicadores de extracción de carne y médula, de acuerdo con la presencia y ubicación de marcas de corte y percusión. En PQ, el caballo sólo presenta marcas en sus costillas, consistentes en cortes y machacado orientados a separarlas de las vértebras y la extracción de su carne junto con la reducción de su tamaño, posiblemente para introducirlas en recipientes de cocción. En este sitio, los únicos especímenes asignados a vaca son un metapodio, que presenta marcas de corte orientadas a separarlo de las falanges, y un cráneo, con una marca de machacado en su hueso frontal posiblemente producida durante el sacrificio del animal.

En términos generales, al comparar las proporciones de marcas en ambos sitios para el taxón oveja - utilizado debido a su predominio absoluto en ambas muestras - se encuentra que existen diferencias en su abundancia (Tabla 6). De acuerdo a esto, se muestra una mayor abundancia de marcas en $\mathrm{PQ}$, con una notable frecuencia de percusión en este último.

Un último aspecto considerado fue el nivel de fragmentación de la muestra, calculando el índice de fragmentación para los taxones oveja, caballo y guanaco, si bien en PQ sólo se calculó el de la oveja debido al escaso tamaño de muestra de los otros taxones. El índice arrojó resultados de 1,81 para la oveja, de 1,42 para el caballo y 1,52 para el guanaco en PY. Para la oveja de PQ, el índice arrojó un resultado de 2,39. Dichas cifras indican, en general, una baja fragmentación, aunque con diferencias entre taxones y sitios. En este sentido, se destaca una mayor fragmentación en PQ que en PY. En cuanto a las diferencias entre taxones de PY, estas indican que la oveja es la que presenta valores más elevados, seguida por el caballo y el guanaco, si bien dentro de un bajo nivel general de fragmentación.

En cuanto a los perfiles de representación de partes, se aplicó el test de Spearman a un nivel de significación del 0,05 a los perfiles anatómicos de la oveja, el guanaco y el caballo de PY y la oveja de PQ (Tabla 7). Dicho test buscó relacionar el MAU\% de estos taxones con los índices de utilidad propuestos para las distintas especies: el de carne, médula, utilidad general y de secado de carne. En la mayoría de los índices empleados relevados no existió una correlación significativa con los perfiles anatómicos. Esta situación es la 


\begin{tabular}{|c|c|c|c|c|c|}
\hline 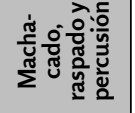 & $\circ$ & $\circ$ & $\circ$ & $\underset{0}{\mathbb{3}}$ & $\circ$ \\
\hline 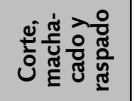 & $\circ$ & $\circ$ & $\circ$ & $\underset{0}{3}$ & $\circ$ \\
\hline 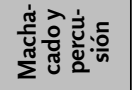 & $\circ$ & $\underset{\mathfrak{F}}{\stackrel{乛}{f}}$ & $\circ$ & $\circ$ & $\circ$ \\
\hline 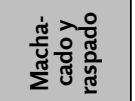 & $\underset{0}{3}$ & 0 & $\circ$ & 0 & $\circ$ \\
\hline 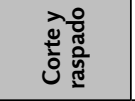 & $\underset{\substack{\widehat{N} \\
\infty \\
0}}{0}$ & $\underset{\widetilde{F}}{\widehat{G}}$ & 0 & $\begin{array}{l}\overline{\vec{n}} \\
\vec{m}\end{array}$ & $\circ$ \\
\hline 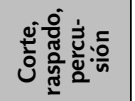 & $\frac{\substack{3 \\
0 \\
0}}{0}$ & $\circ$ & 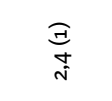 & $\begin{array}{l}\text { i } \\
\text { J }\end{array}$ & $\circ$ \\
\hline 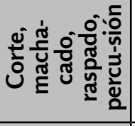 & 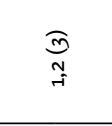 & $\circ$ & 0 & $\circ$ & $\circ$ \\
\hline 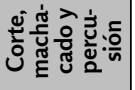 & 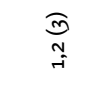 & 0 & $\circ$ & 0 & $\circ$ \\
\hline 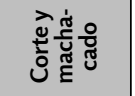 & $\underset{\hat{n}}{\hat{n}}$ & $\underset{\mathfrak{F}}{\stackrel{\Xi}{f}}$ & 0 & 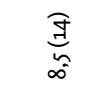 & 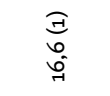 \\
\hline 竎: & $\begin{array}{l}\text { J } \\
6 \\
\text { i }\end{array}$ & $\underset{\Re}{\stackrel{\Xi}{F}}$ & 0 & 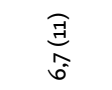 & $\begin{array}{l}\widehat{\Xi} \\
6 \\
0 \\
0 \\
0\end{array}$ \\
\hline 赵:흔 & $\begin{array}{l}\text { J } \\
6 \\
-i\end{array}$ & $\circ$ & $\begin{array}{l}\widehat{N} \\
\infty \\
\text { f }\end{array}$ & $\begin{array}{l}\text { जa } \\
\vec{n} \\
m\end{array}$ & 0 \\
\hline 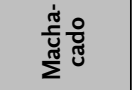 & $\underset{\substack{\widehat{N} \\
\infty \\
0 \\
0}}{ }$ & $\begin{array}{l}\widehat{N} \\
\stackrel{-1}{-1} \\
-1\end{array}$ & $\underset{\substack{\widehat{N} \\
\infty \\
\text { f }}}{ }$ & 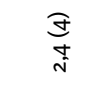 & 0 \\
\hline 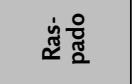 & $\begin{array}{l}3 \\
\text { I } \\
0\end{array}$ & $\circ$ & $\circ$ & 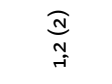 & 0 \\
\hline ัํํ & $\begin{array}{l}\text { 突 } \\
\text { à } \\
\text { ồ }\end{array}$ & 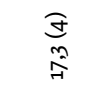 & $\begin{array}{l}\hat{a} \\
a \\
\vec{j}\end{array}$ & $\begin{array}{l}\widehat{\sigma} \\
\stackrel{0}{0} \\
\bar{N}\end{array}$ & 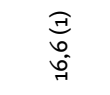 \\
\hline 离 & 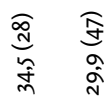 & 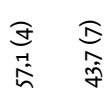 & 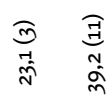 & 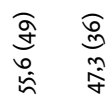 & 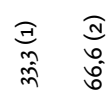 \\
\hline :0 & $\dot{x} \quad \dot{8}$ & $\dot{x} \quad \dot{8}$ & 文 & 希 这 & $\dot{x} \quad \dot{8}$ \\
\hline 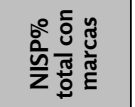 & $\begin{array}{l}\text { जo } \\
a \\
\text { oे }\end{array}$ & 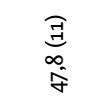 & 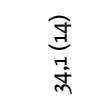 & 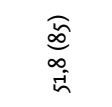 & స్ \\
\hline 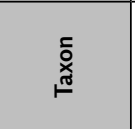 & 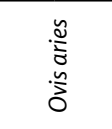 & $\frac{\mathfrak{y}}{\frac{\mathfrak{z}}{w}}$ & 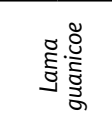 & 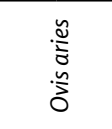 & 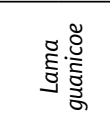 \\
\hline 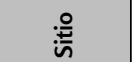 & \multicolumn{3}{|c|}{ ¿ } & \multicolumn{2}{|c|}{$d$} \\
\hline
\end{tabular}

Tabla 6. Marcas de procesamiento en los taxones más representados de ambos sitios, el primer número indica el porcentaje, el número entre paréntesis indica el $N$. En la tercera y quinta columnas se consideran todos los especímenes con marcas sobre el total identificado. Referencias: Ax.: axial, Ap.: apendicular. 


\begin{tabular}{|c|c|c|c|c|c|}
\cline { 3 - 6 } \multicolumn{2}{|c|}{} & Carne & Médula & Secado & $\begin{array}{c}\text { Utilidad } \\
\text { general }\end{array}$ \\
\hline \multirow{3}{*}{ PY } & \multirow{3}{*}{ Ovis aries } & $r=0,25$ & $r=0,31$ & $r=-0,008$ & $r=0,26$ \\
& \multirow{3}{*}{ Equus ferus } & $p=0,31$ & $p=0,21$ & $p=0,9$ & $p=0,22$ \\
\cline { 3 - 7 } & & $p=0,31$ & $r=0,57$ & $r=-0,75$ & $r=-0,36$ \\
& \multirow{3}{*}{ Lama guanicoe } & $r=-0,19$ & $r=0,24$ & $r=-0,82$ & $r=0,31$ \\
& & $p=0,52$ & $p=0,61$ & $p=0,03$ & $p=0,27$ \\
\hline \multirow{3}{*}{ PQ } & Ovis aries & $r=0,3$ & $r=0,27$ & $r=0,17$ & $r=0,55$ \\
& & $p=0,22$ & $p=0,2$ & $p=0,49$ & $p=0,49$ \\
\hline
\end{tabular}

Tabla 7. Resultados de la correlación entre el MAU\% y los índices de carne, médula, secado y utilidad general.

esperada en una economía pastoril en la que los animales domésticos consumidos no son sometidos a un transporte diferencial de partes determinado por su utilidad, sino que ingresan enteros a los espacios residenciales. Si bien esto es válido para el caso de las especies domésticas, la representación de partes del guanaco en PY muestra una correlación negativa fuerte y significativa con el índice de secado de carne. En este sentido, la especie está casi exclusivamente representada por elementos apendiculares. Tal perfil anatómico inclina a considerar el ingreso al sitio de extremidades, las cuales presentan abundante médula y carne, descartando en el campo el resto de la carcasa. Por otra parte, si bien los elementos pertenecientes a cauquén y liebre resultan muy escasos como para realizar correlaciones con los marcos de referencia de densidad ósea, debe remarcarse que todos los huesos pertenecientes a estos taxones son elementos apendiculares. En el caso de estos taxones, es posible que existan procesos atricionales que generaron la subrepresentación de estos elementos en el registro.

\section{Discusión}

\section{Aspectos tafonómicos}

Las variables analizadas indican una serie de patrones que resultan de interés para comprender la organización adoptada por los grupos que habitaron los puestos. En este sentido, los análisis tafonómicos muestran patrones naturales y culturales que afectaron la formación de los sitios, los cuales se relacionan con las estrategias de explotación de los recursos.

De esta forma, las evidencias de ambos puestos apuntan a una frecuencia considerable de los daños producidos por carnívoros en las muestras. Tal evidencia contrasta con la información existente para los carnívoros nativos de Patagonia, que indica que los mismos poseen una baja capacidad de daño (Borrero y Martin, 1996; Fernández, Cruz y Forlano, 2010; Martin, 2006; entre otros). La amplitud, diversidad e intensidad de los efectos relevados se encuentran en marcado contraste con los descriptos para momentos previos al contacto europeo en la región (De Nigris, 2004; Dellepiane, 2014; Rindel, 2008; Rindel y Agnolin, 2013).

La influencia de los carnívoros en la conformación de los perfiles anatómicos relevados para la oveja coincidiría con una predominancia de diáfisis de huesos largos, una subrepresentación de epífisis proximales y una marcada escasez de elementos axiales (Agnolin y Nuevo Delaunay, 2016). Este patrón resulta muy común en sitios 
afectados por carnívoros (Bunn y Kroll, 1986; Haynes, 1980; Marean y Frey, 1997, entre otros). A pesar de ello, las evidencias de furrows en PQ son escasas y limitadas a una epífisis de húmero, mientras que en PY este tipo de daño es inexistente. Perfiles con similares características definen el registro de una amplia variedad de sitios y períodos temporales, muchos de los cuales carecen de evidencias contundentes de destrucción por parte de carnívoros (e.g. De Nigris, 2004; Dellepiane, 2014; Marean y Frey, 1997, entre otros). Ante esto, surge la posibilidad de que la ausencia de estos elementos se deba a otros agentes. En este sentido, la intensa combustión a la que fueron sometidos los conjuntos pudo destruir parte de estos elementos (Lyman, 1994). Asimismo, en diversos conjuntos óseos de Patagonia previos al contacto se ha planteado que el procesamiento humano pudo haber generado perfiles anatómicos similares con escasos elementos axiales y epífisis de huesos largos (Bourlot, 2009; Fernández, 2010). Estos elementos se habrían procesado intensamente con el objeto de extraer la grasa trabecular almacenada en sus tejidos esponjosos. En los casos de PY y PQ, la combinación de una elevada termoalteración, la presencia moderada de marcas de carnívoros y el procesamiento humano estarían influyendo en la conformación de estos perfiles anatómicos, si bien el rol que cada uno de estos factores tuvo en su formación no habría sido equivalente.

Por otra parte, la actividad de los carnívoros pudo tener un efecto más pronunciado sobre los perfiles esqueletarios de los animales de tamaño pequeño. En este sentido, se debe considerar el carácter mayormente apendicular de los huesos de cauquén y liebre presentes en el sitio PY y la casi total ausencia de estos en PQ (Agnolin y Nuevo Delaunay, 2016), además de la presencia de marcas de carnívoro en un espécimen de liebre. Los huesos frágiles y de tamaño pequeño de especies chicas son usualmente triturados e ingeridos por los carnívoros que actúan sobre sitios arqueológicos, desapareciendo rápidamente de los depósitos (Binford, 1981; Ugan, 2010; Walters, 1984). Esta actividad deja como resultado la supervivencia de los elementos apendiculares, quedando los axiales casi completamente destruidos (Ugan, 2010). Para el caso de los sitios analizados podría estar actuando este proceso.

En este contexto, se comprende que el accionar de los carnívoros podría haber resultado en la destrucción de huesos axiales en el caso de los animales pequeños, mientras que en el taxón oveja serían responsables de daños menores, principalmente vinculados con la alteración de las superficies óseas en forma de punctures. A pesar de ello, en esta última especie tales agentes no han sido los únicos ni los principales en darle forma al perfil observado, estando las actividades humanas posiblemente involucradas en la creación de este patrón.

Por último, se debe remarcar que la presencia de marcas de carnívoros en el registro constituye un aspecto distintivo de estos contextos, que los diferencia de los de momentos previos al contacto europeo (Rindel y Agnolin, 2013). De este modo, si bien los sitios difieren en los porcentajes de especímenes afectados por este tipo de daño, ambos indican la existencia de una mayor intensidad y diversidad de daño en momentos históricos. En el caso de PY, éste posee similar porcentaje de marcas que algunos de los sitios prehistóricos de sectores altos, como los sitios Alero Dirección Obligatoria y Alero Gorra de Vasco, los que presentan un 7\% y 9\%, respectivamente (Rindel, 2008). A pesar de ello, en los sitios analizados el tipo de modificaciones óseas registradas resulta más variado, mientras que en los sitios previos al contacto europeo se ve limitada a punctures y scoring. En cuanto a las diferencias entre las muestras, la mayor abundancia de marcas en PQ puede estar vinculada con una mayor presión sobre los recursos a los que accedían los carnívoros. En este sentido, una disminución de la fauna consumible en los alrededores del puesto y la región en general o una elevada abundancia de carnívoros competidores podrían explicar estas diferencias (Blumenschine y Marean, 1993; Faith, Marean y Behrensmeyer, 2007). 
Los datos referentes a la presencia de perros domésticos son coincidentes con las expectativas generadas para una economía pastoril extensiva que emplea grandes extensiones de tierra (Esqueda Coronado y Gutiérrez Ronquillo, 2009). En este contexto, el perro resulta fundamental como herramienta de trabajo, contribuyendo al manejo de la hacienda. Asimismo, su uso en prácticas de caza debió haber resultado esencial para los grupos locales, de acuerdo a lo conocido para momentos históricos (Musters, 2005 y Onelli, 1904).

En cuanto a la incidencia del fuego, se propone que su elevada frecuencia se debería a la incineración de los restos óseos una vez aprovechados como una forma de manejo de los residuos. A favor de esta interpretación se encuentra el hecho de que los huesos calcinados presentan este atributo en toda su superficie, incluyendo en los fragmentos de hueso largo, su canal medular y las superficies de fractura, lo que indica que se encontraron sometidos al fuego luego de procesados. La utilización del fuego en la eliminación de residuos es una práctica muy extendida actualmente en la región y ampliamente registrada en contextos sedentarios a través de todo el mundo (entre otros véase Aguerre, 2000; Moore, Bruno, Capriles y Hastorf, 2010; Tuma, 2006). En contextos sedentarios como el que nos ocupa, la acumulación de residuos orgánicos resulta desventajosa, dado que implica la atracción de insectos y roedores y actúa como un foco de atracción para carnívoros (zorros y pumas) que resultaría en un elemento nocivo para una economía pastoril. En ese contexto, la implementación de tácticas de manejo de residuos se encuentra en consonancia con las nuevas necesidades impuestas por el sedentarismo y la crianza de animales.

Por otra parte, al comparar ambos conjuntos con los procedentes de sitios de momentos previos al contacto y caracterizados por una economía cazadora-recolectora, surge un interesante paralelismo. Los mismos indican que elevadas frecuencias de termoalteración (mayores al 30\% del NISP) se encuentran en cuevas y aleros, siendo muy bajos en los sitios a cielo abierto (Rindel, 2008). Parte de la explicación a este patrón se encontraría en el carácter circunscripto del espacio tanto en los aleros como en los puestos, algo que no se da en el caso de los sitios a cielo abierto. En estos últimos, la disponibilidad de espacio y la consiguiente posibilidad de mover los campamentos una vez que los residuos se convierten en un estorbo, hacen innecesaria la quema de los residuos. Esta práctica sí se explica en espacios circunscriptos en los que las limitaciones impuestas al espacio y la descomposición del material faunístico hacen deseable su eliminación periódica mediante la combustión de los restos.

Otro de los aspectos en los que habría incidido el fuego es en el bajo nivel de identificabilidad detectado en el conjunto, el cual se encuentra muy por debajo de la mayoría de los sitios publicados de Patagonia meridional (Dellepiane, 2014; Fernández, 2010; Mengoni Goñalons, 1999; Rindel, 2008). En este sentido, estudios experimentales indican que la exposición de los huesos a altas temperaturas genera la desaparición de las fibras de colágeno en su estructura, lo que los hace más quebradizos y fragmentarios (Costamagno et al., 1998, 2006; Lyman, 1994; Shipman, Foster y Schoeninger, 1984; Stiner et al., 1995). La marcada diferencia que presentan en su nivel de identificabilidad los huesos termoalterados en contraste con los no afectados por el fuego apoya esta interpretación.

\section{Patrones de procesamiento}

En cuanto a los patrones de procesamiento relevados, los mismos muestran una substancial diversidad de marcas. Estas indican el uso de una importante variedad de recursos faunísticos, posiblemente con un aprovechamiento de una mayor diversidad de especies silvestres durante la ocupación de PY. Asimismo, se observa en ambos conjuntos un especial énfasis en la obtención de carne y médula ósea y la segmentación de partes para su ajuste a los recipientes de cocción. 
Estas evidencias también resultan indicadores de una serie de diferencias entre ambos sitios, principalmente la mayor abundancia de marcas de procesamiento en PQ, acompañada de la aparición de marcas en otros elementos óseos (mandíbulas) y el significativo aumento en el índice de fragmentación. En el caso de las mandíbulas, éstas han sido consideradas por varios autores como fuentes marginales de grasas, cuya utilización se vincula con situaciones de stress alimenticio (Binford, 1981). La evidencia registrada indica un procesamiento más intensivo de las carcasas, con un énfasis en la obtención de grasa. Este cambio se encuentra acompañado por toda una serie de alteraciones en el uso de la fauna, las cuales apuntan al desarrollo de una economía más centrada en la cría especializada de ovinos y orientada a la obtención de carne y lana para el comercio, complementada con prácticas horticultoras y caza (Agnolin y Nuevo Delaunay, 2016). En este marco, la ocupación de PQ en los momentos de avance de las estancias en la región afectó las estrategias empleadas por sus ocupantes, los cuales se encontraron en un contexto de menor disponibilidad de tierras y mayor deterioro ambiental que los que habitaron PY (Goñi y Nuevo Delaunay, 2009; Nuevo Delaunay, 2007, 2008, 2012).

Otro aspecto interesante es el empleo del guanaco en los puestos, dado que los datos registrados muestran el fileteado de su carne y la fractura de sus huesos largos para obtener la médula. Dichas características indican que esta especie formó parte de la dieta de los ocupantes de los sitios, si bien con un rol secundario, el cual pudo haber sido algo más importante en PY (Agnolin y Nuevo Delaunay, 2016). En contraste, actualmente en la región, el uso del guanaco se encuentra mayormente vinculado con su empleo como alimento para perros, con el uso ocasional de su carne para consumo humano. A pesar de esto, los restos de guanaco analizados presentan una escasa proporción afectada por carnívoros (12,1\% en PY y ninguno en PQ), lo cual apoya la idea de su uso principalmente como alimento humano ${ }^{1}$. Por otra parte, un aspecto de interés sobre este taxón es la ausencia de indicadores de la obtención de sus cueros, más si tomamos en cuenta la abundante muestra de raspadores de vidrio hallados en los sitios (Nuevo Delaunay, 2007). Esta ausencia contrasta con lo descripto por numerosos etnógrafos para principios y mediados del siglo XX, cuando parte importante de la economía de los grupos indígenas se basaba en el comercio de pieles y la elaboración de quillangos, confeccionados especialmente con pieles de chulengos (guanacos con menos de 1 mes de edad) (Aguerre, 2000; Caviglia, 2003; Martinic, 1995; Priegue, 2007; Rodríguez, 2010). En este contexto, dicha ausencia es difícil de explicar y podría vincularse, como en el caso de las ovejas, a su comercio con los autopodios aún adheridos (Agnolin y Nuevo Delaunay, 2016). Si este fuera el caso, los cueros serían obtenidos y no se los separaría de los autopodios, por lo que su venta posterior removería estas partes de los sitios y no dejaría indicios de su obtención. Otra posibilidad es que los cueros se extrajesen a los animales pero que se transportasen sin huesos ni carne adheridos, dejando las evidencias de su obtención en el lugar de caza. Asociado a esto, está la notable ausencia de huesos de chulengo en las muestras (Tabla 2), tratándose en casi todos los casos de huesos fusionados, a los que se suman unos pocos no fusionados en PY. Estos últimos consisten en centros que fusionan a partir de los 24-36 meses después de la parición (Kaufmann, 2009). Dicho perfil etario contrasta con lo esperado para una economía que incorpora intensamente el comercio de cueros de chulengo. En cualquier caso, los patrones relevados indican el consumo de carne y médula de guanacos adultos, por lo que en el caso de que se obtuviesen y procesasen cueros de individuos juveniles con intenciones de comercio - como lo indica la evidencia tecnológica (Nuevo Delaunay, 2007) - su carne no sería consumida, al menos de manera que resulte en evidencia ósea. El marcado contraste con los sitios pre-contacto de la región, en los cuales resultan frecuentes porcentajes de huesos no fusionados en torno al $20 \mathrm{y}$ $30 \%$ (Rindel, 2008), es un indicio más de los cambios que tuvieron lugar en el uso de la fauna silvestre.
1. Esto no implica que se descarte un uso del guanaco como alimento para perros, sino más bien que el material óseo depositado en los basurales se vinculaba principalmente al consumo humano. Si el guanaco se usó para alimentarlos, es posible que sus restos estén cerca de los lugares donde los perros eran mantenidos, probablemente en alguna estructura periférica de los sitios. 
Un aspecto de interés - y para el cual es difícil proponer una explicación- es la casi total ausencia de elementos con marcado perimetral (limitado a un solo caso dudoso en un radio distal de oveja en PY). Este tipo de marca es muy abundante en los sitios previos al contacto en la región y se la ha vinculado con formas estandarizadas de procesamiento de las carcasas (Bourlot, Rindel y Aragone, 2009; Muñoz y Belardi, 1998). Actualmente en la región el mismo es empleado ocasionalmente por algunos pobladores rurales (D. Rindel, comunicación personal, 2010).

\section{Conclusiones}

Las evidencias relevadas dan cuenta del proceso de reorganización atravesado por los grupos indígenas de la región tras su instalación en un espacio marginal. Estos incorporaron nuevas fuentes de subsistencia y las articularon de diferentes maneras ante el avance de las estancias en la Meseta del Strobel. De este modo, el cambio de estrategias no sólo afectó la diversidad de especies consumidas, su intensidad de procesamiento o su modo de obtención, sino también los factores postdepositacionales que actuaron sobre los conjuntos. En este sentido, las nuevas prácticas configuraron un registro zooarqueológico marcadamente diferente, caracterizado por el predominio de materiales de oveja y caballo combinados con especies silvestres, pero también por la existencia de elevadas proporciones de marcas de carnívoros, termoalteración y la disposición de los materiales en estructuras de descarte. Dicho registro apunta a la existencia de estrategias flexibles de organización de la subsistencia, altamente variables de acuerdo con las condiciones socioambientales.

El desarrollo de estas estrategias encuentra su fin en la región con el abandono de la meseta por parte de los grupos indígenas. Este abandono no se da por la deficiencia de las estrategias implementadas, sino por la aplicación de una nueva legislación que limitó el acceso a las tierras por parte de estos grupos. En este sentido, el uso de amplios espacios sobre los que no se poseía la tenencia legal, parte integral de estas estrategias, se vio impedido (Nuevo Delaunay, 2015). El proceso de provincialización de Santa Cruz implicó la regulación de la tenencia de tierras, restringiendo el acceso a gran parte de las mismas para los grupos que no poseían la propiedad (Nuevo Delaunay, 2015). Desde este punto de vista, el uso de los espacios marginales se habría visto aún más constreñido, lo cual, combinado con la marcada estacionalidad y escasa productividad de las tierras, llevó a un declive final y al abandono de las ocupaciones. Un proceso análogo, combinado con un mercado internacional desfavorable y profundizado por la erupción del volcán Hudson, generó el despoblamiento de la meseta por parte de los ocupantes criollos a fines del siglo XX (Nuevo Delaunay, 2015).

Las estrategias adoptadas por los grupos exploradas en este trabajo arrojan una imagen muy diferente de los grupos tehuelches del siglo XX, los cuales han sido usualmente caracterizados como restos de un pasado condenado a desaparecer por su incapacidad de cambio (Rodríguez, 2010). En su lugar, el registro indica que aún dentro de un contexto de desarticulación social, se dio un proceso de reacomodamiento y cambio en las estrategias, el cual finaliza ante los constreñimientos impuestos por una nueva legislación. Este nuevo panorama condujo a un proceso de abandono de los asentamientos en la meseta del Strobel y la migración a centros urbanos.

\section{Agradecimientos}

A Rafael Goñi y Diego Rindel por los consejos y la paciencia, a Amalia Nuevo Delaunay por los datos sobre los sitios, a mis compañeros: Juan Dellepiane, Francisco Guichón, Josefina Flores Coni, Gisela Cassiodoro y Sebastián Diego Enrique Pasqualini por la 
ayuda en la confección del manuscrito y sus consejos. Las investigaciones realizadas fueron financiadas con fondos de ANPCYT PICT’04 N² 26295, Secretaría de Cultura de la Nación (INAPL) y UBACYT n ${ }^{\circ}$ F065. Agradezco asimismo a los evaluadores, cuyos comentarios contribuyeron a mejorar substancialmente este manuscrito. 


\section{Q Referencias citadas}

"Abe, Y. (2005). Hunting and Butchery patterns of the Evenki in northern Transbaikalia, Russia. (Tesis Doctoral inédita), Stony Brook University, EEUU.

"Agnolin, A. M. y Nuevo Delaunay, A. (2016). Arrinconamiento y marginalidad durante el siglo XX: la evidencia arqueofaunística de los sitios Puesto Yatel y Puesto Quintillán (centro oeste de la Pcia. de Santa Cruz, Patagonia argentina). En F. Mena (Ed.), De Mar a Mar. Actas de las IX Jornadas de Arqueología de la Patagonia (pp. 381-389) Santiago: Ediciones CIEP / Ñire Negro.

"Aguerre, A. M. (2000). Las vidas de Pati en la toldería tehuelche del río Pinturas y el después. Buenos Aires: Facultad Filosofía y Letras, Universidad de Buenos Aires.

» Andrés, M., Gidna, A. O., Yravedra, J. y Domínguez-Rodrigo, M. (2012). A study of dimensional differences of tooth marks (pits and scores) on bones modified by small and large carnivores. Archaeological and Anthropological Sciences, 4(3), 209-219.

»Asmussen, B. (2009). Intentional or incidental thermal modification? Analyzing site occupation via burned bone. Journal of Archaeological Science, 36, 528-536.

》Bandieri, S. (2005). Historia de la Patagonia. Buenos Aires: Sudamericana.

" Barbería, E. M. (1987). El avance de la ganadería ovina y el indígena de Santa Cruz. Mundo Ameghiniano, 7, 19-53.

" Barbería, E. M. (1996). Los dueños de la tierra en la Patagonia Austral, 1880-1920. Santa Cruz: Universidad Nacional de la Patagonia Austral.

"Behrensmeyer, A. K. (1978). Taphonomic and ecological information from bone weathering. Paleobiology, 4, 150-162.

» Binford, L. R. (1978). Nunamiut Ethnoarchaeology. Nueva York: Academic Press.

» Binford, L. R. (1981). Bones: Ancient Men and Modern Myths. Nueva York: Academic Press.

» Blumenschine, R. J. y Marean, C. W. (1993). A Carnivore's View of Archaeological Bone Assemblages. En J. Hudson (Ed.), From Bones to Behavior (pp. 273-300). Carbondale: Southern Illinois Press.

»Borrero, L. A. (1990). Taphonomy of Guanaco Bones in Tierra del Fuego. Quaternary Research, 34, 361-371.

" Borrero, L. A. y Martín, F. M. (1996). Tafonomía de carnívoros: un enfoque regional. En J. Gómez Otero (Ed.), Arqueología: Solo Patagonia (pp. 189-198). Puerto Madryn: Centro Nacional Patagónico (CONICET).

» Bourlot, T. (2009). Zooarqueología de sitios a cielo abierto en el lago Cardiel, provincia de Santa Cruz: Fragmentación ósea y consumo de grasa animal en grupos cazadoresrecolectores del Holoceno tardío. (Tesis Doctoral Inédita), Universidad de Buenos Aires, Argentina.

» Bourlot, T., Rindel, D. y Aragone, A. (2009). La fractura transversa / marcado perimetral en sitios a cielo abierto durante el Holoceno Tardío en el Noroeste se Santa Cruz. En M. Salemme, F. Santiago, M. Álvarez, E. Piana, M, Vázquez y E. Mansur (Eds.), Arqueología de la Patagonia: una mirada desde el último confín (pp. 693-706). Ushuaia: Editorial Utopías.

» Bunn, H. T. y Kroll, E. M. (1986). Systematic butchery by Plio-Pleistocene hominids at Olduvai Gorge, Tanzania. Current Anthropology, 27(5), 431-442. 
»Casamiquela, R., Martinic, M., Mondelo, O. y Perea, E. (1991). Del Mito a la realidad. Evolución iconográfica del pueblo tehuelche meridional. Viedma: Fundación Ameghino.

»Caviglia, S. (2003). El arte de las mujeres Aónik'enk y Gününa küna-kay guaj'enk o kay gütrruj (las capas pintadas). Relaciones de la Sociedad Argentina de Antropología, XXVII, 41-74.

» Costamagno, S., Griggo, C. y Mourre, V. (1998). Approche expérimentale d'un problème taphonomique: utilisation de combustible osseux au Paléolithique. Préhistoire Européenne, 13, 167-194.

» Costamagno, S., Thery-Parisot, I., Brugal, J. P. y Guibert, R. (2006). Taphonomic consequences of the use of bones as fuel. Experimental data and archaeological applications. En T. O'Connor (Ed.), Biosphere to Lithosphere. New studies in vertebrate taphonomy (pp. 51-62). Oxford: Oxbow Books.

»De Nigris, M. (2004). El consumo en grupos cazadores recolectores. Un ejemplo zooarqueológico de Patagonia meridional. Buenos Aires: Sociedad Argentina de Antropología.

»De Nigris, M. y Mengoni Goñalons, G. (2005). The guanaco as a source of meat and fat in the Southern Andes. En J. Mulville y A. Outram (Eds.), The zooarchaeology offats, oils and dairying (pp. 160-166). Oxford: Oxbow Books.

»Dellepiane, J. M. (2014). Zooarqueología de espacios mesetarios. Patrones de subsistencia y obtención de recursos en el centro oeste de Santa Cruz durante el Holoceno tardío. (Tesis de Licenciatura inédita), Universidad del Centro de la Ciudad de Buenos Aires, Argentina.

»Domínguez-Rodrigo, M. y Piqueras, A. (2003). The use of tooth pits to identify carnivore taxa in toothmarked archaeofaunas and their relevance to reconstruct hominid carcass processing behaviours. Journal of Archaeological Science, 30, 1385-1391.

»Esqueda Coronado, M. H. y Gutiérrez Ronquillo, E. (2009). Producción de ovinos de pelo bajo condiciones de pastoreo extensivo en el norte de México. México: Inifap.

» Faith, J. T., Marean, C. W. y Behrensmeyer, A. K. (2007). Carnivore competition, bone destruction, and bone density. Journal of Archaeological Science, 34(12), 2025-2034.

»Fernández, P. M. (2010). Cazadores y presas. 3500 años de interacción entre seres humanos y animales en el noroeste de Chubut. Buenos Aires: Fundación de Historia Natural Félix de Azara.

»Fernández, P. M., Cruz, I. y Forlano, A. (2010). Sitio 37: una madriguera de carnívoro en el norte de la Patagonia Andina (Cholila, Provincia de Chubut, Argentina). En M. A. Gutiérrez, M. De Nigris, P. M. Fernández, M. Giardina, A. F. Gil, A. Izeta, G. Neme y H. D. Yacobaccio, (Eds.), Zooarqueología a principios del siglo XXI: aportes teóricos, metodológicos y casos de estudio (pp. 409-417). Buenos Aires: Libros del Espinillo.

» Fisher, J. W. (1995). Bone surface modifications in zooarchaeology. Journal of Archaeological Method and Theory, 2, 7-68.

» Gifford-Gonzalez, D. (1989). Ethnographic analogues for interpreting modified bones: some cases from east Africa. En R. Bonnichsen y M. Sorg (Eds.), Bone Modification (pp. 179-246). Orono: Center for the Study of the First Americans, University of Maine.

»Goñi, R. y Nuevo Delaunay, A. (2009). La Arqueología como "fuente” de la Historia. En M. Salemme, F. Santiago, M. Álvarez, E. Piana, M. Vázquez y E. Mansur (Eds.), Arqueología de la Patagonia. Una mirada desde el último confín (pp. 149-158). Ushuaia: Utopías.

» Hatcher, J. B. (1903). Narrative of the Expeditions. Geography of Southern Patagonia. Reports of the Princeton University Expeditions to Patagonia, 1896-1899, 1, 1-314. 
" Haynes, G. (1980). Prey bones and predators: potential ecologic information from analysis of bone sites. Ossa, 7, 75-97.

» Ioannidou, E. (2003). Taphonomy of animal bones: species, sex, age and breed variability of sheep, cattle and pig bone density. Journal of Archaeological Science, 30, 355-365.

» Kaufmann, C. A. (2009). Estructura de edad y sexo en guanaco. Estudios actualísticos y arqueológicos en Pampa y Patagonia. Buenos Aires: Sociedad Argentina de Antropología.

»Kent, S. (1993). Variability in faunal assemblages: the influence of hunting skill, sharing, dogs and mode of cooking on faunal remains at a sedentary Kalahari community. Journal of Anthropological Archaeology, 12, 323-385.

» Klein, R. G. (1976). The Mammalian Fauna of the Klasies River Mouth Sites, Southern Cape Province, South Africa. South African Archaeological Bulletin, 31, 75-98.

» Lam, L. Y., Pearson, O. M., Marean, C. W. y Chen, X. (2003). Bone density studies in zooarchaeology. Journal of Archaeological Science, 30, 1701-1708.

» Lam, L. Y., Xinghin Chen, M. y Pearson, O. (1999). Intertaxonomic variability in patterns of bone density and the differential representation of bovid, cervid and equid elements in the archaeological record. American Antiquity, 64, 343-362.

» Lyman, R. L. (1994). Vertebrate Taphonomy. Cambridge: Cambridge University Press.

" Marean, C. W. y Frey, C. J. (1997). The bones from caves to cities: Reverse utility curves as methodological artifacts. American Antiquity, 62, 698-711.

»Martin, F. M. (2006). Carnívoros y Huesos Humanos de Fuego-Patagonia: Aportes desde la Tafonomía Forense. Buenos Aires: Sociedad Argentina de Antropología.

» Martinic, M. (1995). Los Aónikenk. Historia y Cultura. Punta Arenas: Ediciones de la Universidad de Magallanes.

» Mengoni Goñalons, G. L. (1999). Cazadores de guanacos de la estepa patagónica. Buenos Aires: Sociedad Argentina de Antropología.

» Morello, J. H., Matteucci, S. D., Rodríguez, A. F. y Silva, M. E. (2012). Ecorregiones y Complejos Ecosistémicos Argentinos. Buenos Aires: Orientación Gráfica Editora.

» Moore, K., Bruno, M., Capriles, J. M. y Hastorf, C. (2010). Integrated contextual approaches to understanding past activities using plant and animal remains from Kala Uyuni, Lake Titicaca, Bolivia. En A. M. VanDerwarker y T. M. Peres (Eds.), Integrating Zooarchaeology and Paleoethnobotany: A consideration of issues, methods, and cases (pp. 173-203). Dordrecht: Springer.

" Muñoz, S. y Belardi, J. B. (1998). El marcado perimetral en los huesos largos de guanaco Cañadón Leona (Colección Junius Bird): implicaciones arqueofaunísticas para la Patagonia meridional. Anales del Instituto de la Patagonia. Serie Ciencias Históricas, 26, 107-118.

" Musters, G. C. (2005). Vida entre los Patagones. Buenos Aires: El Elefante Blanco.

»Nuevo Delaunay, A. (2007). Tecnología vítrea en el siglo XX, Lago Strobel (Santa Cruz, Argentina). En F. Morello, M. Martinic, A. Prieto y G. Bahamonde (Eds.), Arqueología de Fuego-Patagonia. Levantando piedras, desenterrando huesos...y develando arcanos (pp. 853-859). Punta Arenas: Ediciones CEQUA.

"Nuevo Delaunay, A. (2008). Arqueología del Siglo XX: dos casos de estudio en la cuenca del Lago Strobel. (Tesis de Licenciatura inédita), Universidad de Buenos Aires, Argentina.

» Nuevo Delaunay, A. (2012). Disarticulation of Aónikenk Hunter-Gatherer Lifeways during the Late-19th and Early-2oth Centuries: Two Case Studies from Argentinean Patagonia. Historical Archaeology, 46(3), 149-164. 
» Nuevo Delaunay, A. (2013). Tecnología lítica y asentamientos modernos: análisis de un conjunto de bolas líticas del siglo XX. En A. F. Zangrando y R. Barberena (Eds.), Tendencias teórico-metodológicas y casos de estudio en la arqueología de la Patagonia (pp. 469-474). San Rafael: Museo de Historia Natural de San Rafael.

» Nuevo Delaunay, A. (2015). Transformación del paisaje arqueológico rural en el centrooeste de la provincia de Santa Cruz, siglo XX. (Tesis Doctoral inédita), Universidad de Buenos Aires, Argentina.

» Nuevo Delaunay, A., Goñi, R. A., Jimenez, N. L. y Cecuk, L. (2014). Marginalidad y adecuación en el siglo XX: dos casos de estudio en la cuenca del lago Strobel. En R. A. Goñi, J. B. Belardi, G. Cassiodoro y A. Re (Eds.), Arqueología de las cuencas de los lagos Cardiel y Strobel. Poblamiento Humano y Paleoambientes en Patagonia (pp. 134-156). Buenos Aires: Aspha Ediciones.

» Nuevo Freire, C. y Vázquez Mercerat, A. (2014). Las “zonas” Lago Cardiel y Lago Strobel: algunas consideraciones sobre el poblamiento ganadero inicial. En R. Goñi, J. B. Belardi, G. Cassiodoro y A. Re (Eds.), Arqueología de las cuencas de los lagos Cardiel y Strobel. Poblamiento humano y paleoambientes en Patagonia (pp. 178-198). Buenos Aires: Aspha Ediciones.

» Oliva, G., González, L., Rial, C. y Livraghi, E. (2001). El ambiente en la Patagonia Austral. En P. Borrelli y G. Oliva (Eds.), Ganadería Ovina sustentable en la Patagonia austral. Tecnología de Manejo Extensivo (pp. 19-83). Buenos Aires: PRODESAR.

» Onelli, C. (1904). Trepando los Andes. Buenos Aires: Compañía Sudamericana de Billetes de Banco.

» Outram, A. y Rowley-Conwy, P. (1998). Meat and Marrow Utility Indices for Horse (Equus). Journal of Archaeological Science, 25, 839-849.

» Potts, R. y Shipman, P. (1981). Cut-marks made by stone tools on bones from Olduvai Gorge, Tanzania. Nature, 291, 577-580.

»Priegue, C. N. (2007). En memoria de los Abuelos. Historia de Vida de Luisa Pascual, Tehuelche. Bahía Blanca: Publitek.

»Ramos, V. (1982). Geología de la región del lago Cardiel, Provincia de Santa Cruz. Revista Asociación Geológica Argentina, 37(1), 23-49.

»Rindel, D. (2008). Arqueología de momentos tardíos en el noroeste de la Provincia de Santa Cruz (Argentina): una perspectiva faunística. (Tesis Doctoral inédita), Universidad de Buenos Aires, Argentina.

» Rindel, D. y Agnolin, A. (2013). Carnívoros y registro arqueológico en Patagonia Meridional: una relación cambiante a lo largo del tiempo. Trabajo presentado en el III Congreso Nacional de Zooarqueología, Tilcara, Jujuy.

»Rodríguez, M. (2010). De la "extinción" a la autoafirmación: procesos de visibilización de la Comunidad Tehuelche Camusu Aike (provincia de Santa Cruz, Argentina). (Tesis Doctoral inédita), Georgetown University, EEUU.

»Shipman, P., Foster, G. y Schoeninger, M. (1984). Burnt bones and teeth: An experimental study of color, morphology, crystal structure and shrinkage. Journal of Archaeological Science, 11, 307-325.

»Stahl, P. W. (1999). Structural density of domesticated South American camelid skeletal elements and the archaeological investigation of prehistoric Andean ch'arki. Journal of Archaeological Science, 26, 1347-1368. 
» Stiner, M. C., Kuhn, S. L., Weiner, S. y Bar-Yosef, O. (1995). Differential burning, recrystallization, and fragmentation of archaeological bone. Journal of Archaeological Science, 22, 223-237.

"Symmons, R. (2005). New density data for unfused and fused sheep bones, and a preliminary discussion on the modeling of taphonomic bias in archaeofaunal age profiles. Journal of Archaeological Science, 32, 1691-1698.

» Théry-Parisot, I. (2002). Fuel management (bone and wood) during the lower Aurignacian in the Pataud rock shelter (Lower Palaeolithic, Les Eyzies de Tayac, Dordogne, France): contribution of experimentation and anthraco-analysis to the study of the socioeconomic behaviour. Journal of Archaeological Science, 29, 1415-1421.

» Thery-Parisot, I., Brugal, J. P., Costamagno, S. y Guilbert, R. (2004). Conséquences taphonomiques de l'utilisation des ossements comme combustible. Approche expérimentale. Les nouvelles de L'archéologie, 95, 19-22.

»Tuma, M. W. (2006). Ethnoarchaeology of Subsistence Behaviors within a Rural African American Community: Implications for Interpreting Vertebrate Faunal Data from Slave Quarters Areas of Antebellum Plantation Sites. Historical Archaeology, 40(4), 1-26.

» Ugan, A. (2010). The effect of cooking on the survivorship of jackrabbit skeletons (Lepus californicus) presented to desert scavengers of the eastern Great Basin, North America. International Journal of Osteoarchaeology, 20, 214-226.

"Walters, I. (1984). Gone to the Dogs: a study of bone attrition at a Central Australian campsite. Mankind, 14(5), 389-40.

»Walters, I. (1988). Fire and bones: patterns of discard. En B. Meehan y R. Jones (Eds.), Archaeology with Ethnography: an Australian Perspective (pp. 215-221). Canberra: Australian National University. 